\title{
ORBITAL PERIOD VARIATION AND MORPHOLOGICAL LIGHT CURVE STUDIES FOR THE W UMa BINARY BB PEGASI
}

\author{
Magdy A. Hanna and Nabil S. Awadalla \\ National Research Institute of Astronomy and Geophysics (NRIAG), Helwan, Cairo, EGYPT \\ (Received December 20, 2010; Revised March 03, 2011; Accepted May 16, 2011)
}

\begin{abstract}
The photometric light curves of the W-type W UMa eclipsing contact binary system BB Pegasi have been found to be extremely asymmetric over all the observed 63 years in all wavelengths UBVR. The light curves have been characterized by occultation primary minima. Hence, the morphology of these light curves has been studied in view of these different asymmetric degrees. The system shows a distinct O'Connell effect, as well as depth variation. A 22.96 years of stellar dark spots cycle has been determined for the system. Almost the same cycle $(22.78 \mathrm{yr})$ has been found for the depth variation of MinI and MinII. We also present an analysis of mid-eclipse time measurements of BB Peg. The analysis indicates a period decrement of $5.62 \times 10^{-8}$ day/yr, which can be interpreted in terms of mass transfer at a rate of $-4.38 \times 10^{-8} \mathrm{M}_{\odot} / \mathrm{yr}$, from the more to the less massive component. The $O-C$ diagram shows a damping sine wave covering two different cycles of $17.0 \mathrm{yr}$ and $12.87 \mathrm{yr}$ with amplitudes equal to 0.0071 and 0.0013 day, respectively. These unequal durations show a non-periodicity which may be explained as a result of magnetic activity cycling variations due to star spots. The obtained characteristics are consistent with similar chromospherically active stars, when applying the Applegate's (1992) mechanism.
\end{abstract}

Key words : binaries: close — binaries: eclipsing — stars: individual (BB Peg) — stars: W-subtype W UMa - period variation: magnetic activity.

\section{INTRODUCTION}

The low temperature W UMa contact binary (LTCB) system BB Peg $\left(M_{v}=11.6\right.$, spectral type F8V, $P=$ $\left.0^{d} .361501\right)$ was first classified as a binary by Hoffmeister (1931). Its period was revised by Whitney (1959) to be 0.3615015 day.

The BV observations of Cerruti-Sola et al. (1980) revealed a variable degree of asymmetry from the yellow to the blue light curve, and a phase shift of the secondary minimum. They classified the system as a contact of the $\mathrm{W}$-subtype, with the fill-out parameter of $37 \%$.

Leung et al. (1985) have found the light curves of BB Peg to be very complex, with large asymmetry and significant discrepancy in the differential eclipse depth (Min I and Min II) between the V- and B- light curves. The system has been also found to be in over contact with small degree of $12 \%$. The UBV observations of Awadalla (1988) have shown a variable depth of the primary eclipse that is a total occultation, and an increase of the orbital period.

Zola et al. (2005) have found that the configuration of BB Peg is contact with intermediate $(21 \%)$ fill out factor, using BVR observations at the Mt. Suhora observatory in 2004. They have stated that the O'Connell effect is noticeable for the system. Kalomeni et al. (2007) have confirmed the claim and determined the rate of mass transfer from the less massive to the more massive component. Snyder (2008) has shown that the light curve of the system is asymmetric (O'Connell effect) and displays total annular eclipses in the primary. He has found two small cool stellar spots on the secondary star.

The first radial velocity study of the system has been done by Hrivnak (1990) which gives the spectroscopic value of the mass ratio $q_{s p}=0.34$. Later, it has been redetermined to be $q_{s p}=0.36$ by Lu and Rucinski (1999). D'Angelo et al. (2006) have suggested from their spectroscopic study the existence of an M-type dwarf star of minimum mass $M_{3}=0.18 \mathrm{M}_{\odot}$ orbiting around the binary.

Several photometric solutions of the light curves for the BB Peg system have been determined by various authors; among them Cerruti-Sola et al. (1981), Zola et al. (2005) and Snyder (2008). Kalomeni et al. (2007) have observed the system in $V$ and $R$ during 8 nights between Aug-Dec 2004, and during two nights in 2006. They have determined the absolute parameters for the system, and analyzed the light curves obtained by themselves as well as those obtained by Cerruit-Sola \& Scaltriti (1980), Zhai \& Zhang (1979) and Awadalla (1988). The analysis and fitting light curves for the four sets of the observations have different and poor results. Both sets of light curves for BB Peg obtained by Cerruti-Sola \& Scaltriti (1980) and 


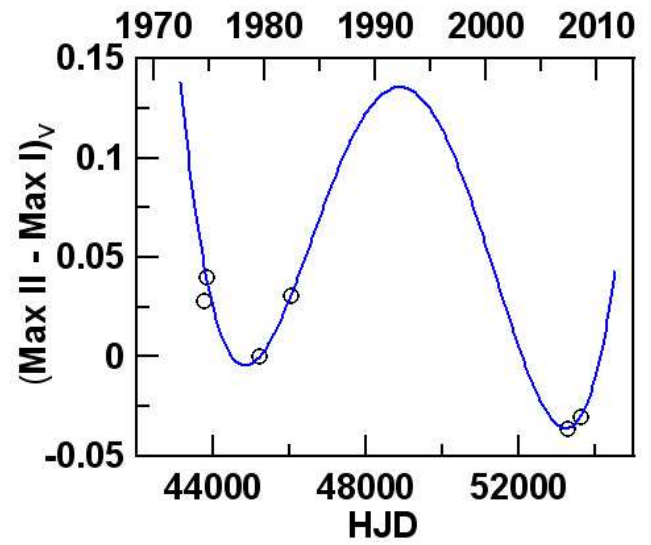

Fig. 1.- Relation between the O'Connell effect (MaxII-MaxI) and the corresponding HJD and years for all the published light curves in the $\mathrm{V}$-filter.

Zhai \& Zhang (1979) show distinct asymmetry at maximum light, even though the two sets were observed in a two-month interval. This means that the light curves for the system BB Peg are undergoing relatively rapid changes. Hence, all the analysis of the light curves for the present system have been poorly determined, and in particular the fill out ratio, the O'Connell effect and the depth differences of minima.

Since most of the adopted solutions for the system BB Peg are poor due to the asymmetry of the light curves, their morphology has to be studied again, especially its asymmetry and the depth difference over all the time interval that the system has been observed. Moreover, the $(O-C)$ diagram has been examined to explain the orbital period behaviour, in view of any possible relation with magnetic activity cycling for the system.

\section{STUDY OF LIGHT CURVE MORPHOLO- GIES}

All the light curves observed from HJD 2449564.0 to HJD 2454359.0 have asymmetric shapes in both maxima and minima in all $U B V R$ filters, except those observed by Cerruti-Sola and Scaltriti (1980) which are in equal depths but slightly differ in maxima -i.e. likely to be symmetric. From the compilation of all published light curves, we can investigate the short time light variation.

Most of the light curves of BB Peg have been analysed by various authors with different methods, such as the Wood method (1972), the Russel \& Merrill method (1952) and the Wilson \& Devinney (W-D) method (1971). Most of the solutions that have been obtained

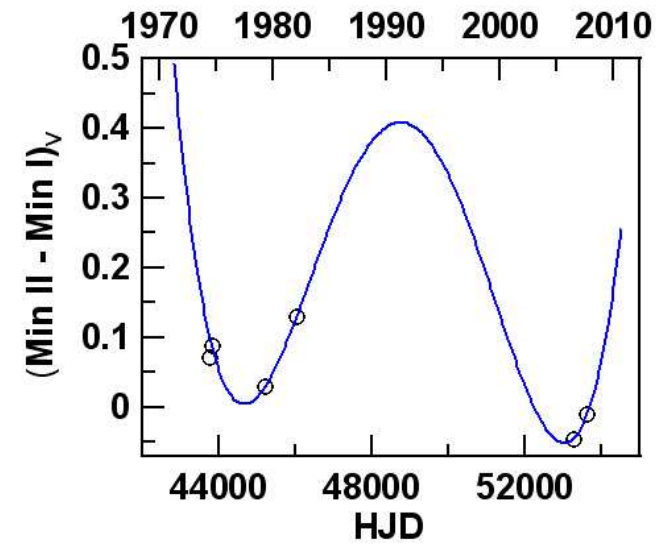

Fig. 2. - Relation between the depth differences (MinII-MinI) and the corresponding HJD and years for all the published light curves in the V-filter.

show poor agreements with data, due to either a variable degree of asymmetry from yellow to blue light curve or a phase shift of the second minimum (CerrutiSola et al. 1981).

Kalomeni et al. (2007) have analyzed (using the W-D code) the light curves observed by Cerruti-Sola and Scaltriti (1980), Zhai and Zhang (1979), Awadalla (1988), as well as their observations simultaneously with Lu and Rucinski's (1999) radial velocities. They have determined different parameters and various fillout factors for the system.

Tables 1 and 2 summarize the maximum difference (O'Connell effect, MaxII-MaxI) and the depth difference (MinII-MinI) in UBVR for all the published light curves with the corresponding observed date (HJD), respectively. Figs. 1 and 2 show the changes in the O'Connell effect and the depth difference for BB Peg with the corresponding mean HJD, respectively. The solid lines represent $4^{\text {th }}$ degree polynomial fits, for both the O'Connell effect and the depth difference. The two figures show nearly periodical cyclic changes with the same behavior. The correlation coefficient and the standard deviation for both polynomials have been determined: $r=0.985, S D=0.012$, and $r=0.989$, $S D=0.021$, respectively. The O'Connell effect shows a periodicity of 22.96 years from HJD 2444903 to 2453290; approximately the same periodicity has been found for the depth differences from HJD 2444645 to 2452968 , i.e. 22.78 years.

The light curve observed by Zola et al. (2005) shows a flat bottom for the primary minimum, and a little shallower secondary minimum. It also shows an inclination equal to $88^{\circ} .5$ and a $21 \%$ fill out-ratio. Kalomeni et al. (2007) have found that the system is over- 
Table 1.

O'Connell effect

\begin{tabular}{llrrrrrr}
\hline \hline Observation Date & HJD. & \multicolumn{2}{c}{ O'Connell Effect $^{\dagger}$ (MaxII-MaxI) } & \multirow{2}{*}{ Ref. } \\
\cline { 3 - 6 } & $2400000+$ & U & B & V & R & \\
\hline & & & & & & & \\
Aug 08/1978- Sep 12/1978 & 43747 & 0.000 & 0.043 & 0.028 & - & \\
Oct 01/1978- Nov 30/1978 & 43811 & - & 0.050 & 0.040 & - & {$[2]$} \\
Aug 26/1982- Aug 27/1982 & 45208 & 0.045 & 0.055 & 0.000 & - & {$[3]$} \\
Nov 18/1984- Nov 22/1984 & 46026 & -0.010 & 0.039 & 0.031 & - & {$[3]$} \\
Aug 10/11/2004 Oct 05-07/2004 & 53256 & - & -0.055 & -0.036 & -0.027 & {$[4]$} \\
Aug-Dec/2004- Oct 2006 & 53613 & - & - & -0.030 & -0.015 & {$[5]$} \\
\hline
\end{tabular}

Ref.: [1] Cerruti-Sola and Scaltriti (1980), [2] Leung et al. (1985), [3] Awadalla (1988), [4] Zola et al. (2005), [5] Kalomeni et al. (2007). † mean value.

Table 2.

Depth difference and fill-out ratio

\begin{tabular}{|c|c|c|c|c|c|c|c|}
\hline \multirow[t]{2}{*}{ Observation Date } & \multirow{2}{*}{$\begin{array}{l}\text { HJD. }^{\dagger} \\
2400000+\end{array}$} & \multicolumn{4}{|c|}{ 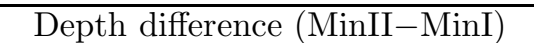 } & \multirow[t]{2}{*}{$\overline{\mathrm{F}-\text { ratio } \%}$} & \multirow[t]{2}{*}{$\overline{\text { Ref. }}$} \\
\hline & & $\mathrm{U}$ & $\mathrm{B}$ & $\mathrm{V}$ & $\mathrm{R}$ & & \\
\hline Aug 08/1978 - Sep 12/1978 & 43747 & 0.112 & 0.057 & 0.071 & - & $35-37$ & [1] \\
\hline Oct $01 / 1978-$ Nov $30 / 1978$ & 43811 & - & 0.081 & 0.088 & - & $12-38$ & {$[2]$} \\
\hline Aug 26/1982 - Aug 27/1982 & 45208 & 0.090 & 0.050 & 0.030 & - & - & [3] \\
\hline Nov 18/1984 - Nov 22/1984 & 46026 & 0.240 & 0.150 & 0.130 & - & 33 & {$[3]$} \\
\hline Aug $10 / 11 / 2004$ Oct $05-07 / 2004$ & 53256 & - & -0.045 & -0.045 & -0.036 & 21 & [4] \\
\hline Aug-Dec/2004- Oct 2006 & 53613 & - & - & -0.010 & 0.000 & 34 & {$[5]$} \\
\hline
\end{tabular}

Ref.: Same as Table $1 . \quad \dagger$ mean value.

Table 3.

Ephemerids of BB Peg by various authors

\begin{tabular}{lccl}
\hline \hline JD.+240000 & Period & Quadratic term & Reference \\
\hline & & & \\
30285.75400 & 0.36150720 & & Whitney (1943) \\
30285.75500 & 0.36150150 & & Whitney (1959) \\
30285.76300 & 0.36150100 & & Zhai \& Zhang (1979) \\
30285.76180 & 0.36150027 & $2.35 \times 10^{-11}$ & Qian (2001) \\
43764.33340 & 0.36150210 & $2.30 \times 10^{-11}$ & Cirruti Sola \& Scatriti (1980) \\
43764.33340 & 0.36105150 & & Cirruti Sola \& Scatriti (1980) \\
50657.45990 & 0.36150150 & & Kalomeni et al. (2007) \\
43764.34160 & 0.36150147 & & Kreiner (2004) \\
52500.03730 & 0.36150256 & $1.49 \times 10^{-11}$ & Snyder (2008) \\
53228.45740 & 0.36150100 & & Zola (2005) \\
43764.34160 & 0.36150147 & & Present work ${ }^{\dagger}$ \\
43764.33810 & 0.36150099 & $1.08 \times 10^{-11}$ & Present work ${ }^{\dagger}$ \\
43764.33319 & 0.36150213 & & Present work \\
43764.33083 & 0.36150295 & $-2.78 \times 10^{-11}$ & Present work \\
\hline
\end{tabular}

$\dagger$ All v, pg, pe and CCD minima times. $\ddagger$ Only pe and CCD minima times. 
Table 4.

Comparison between number of data used in previous and present studies

\begin{tabular}{|c|c|c|c|c|c|}
\hline & Visual & Photog. & $\overline{\mathrm{Pe}}$ & $\overline{\mathrm{CCD}}$ & Total \\
\hline Cerruti-Sola and Scaltriti (1980) & $5^{*}$ & 25 & 5 & - & 35 \\
\hline Awadalla (1988) & 9 & 25 & 9 & - & 43 \\
\hline Qian (2001) & \multicolumn{2}{|r|}{33} & 23 & 4 & 60 \\
\hline Kalomeni et al. (2007) & $7^{* *}$ & 14 & 33 & 19 & 73 \\
\hline Present work & $25^{\dagger}$ & $127^{\dagger}$ & 45 & 45 & 90 \\
\hline
\end{tabular}

* The authors have used only 5 minima out of 9 , due to the data large internal errors; ${ }^{* *}$ The authors have used only 7 out of 9 minima. $\dagger$ The visual and photographic minima times are listed in Table 10 of the Appendix; these have been used only in Fig. 4 and in eq.(3).

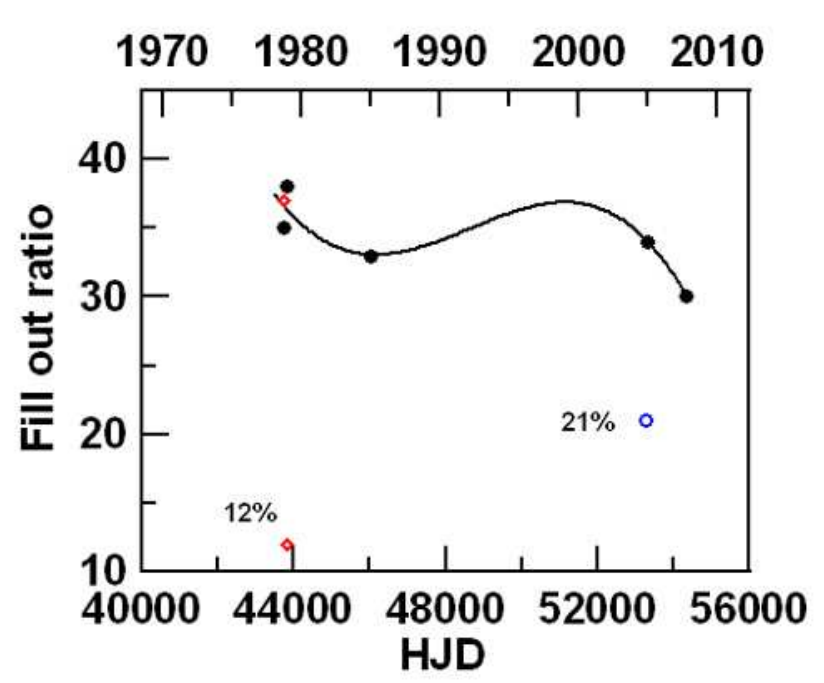

Fig. 3.- Variation of the fill-out ratio, by various authors.

luminous and oversized, like the other W-subtype of W UMa binaries.

Awadalla's (1988) light curves show that the primary minima are deeper than the secondary ones in both sets of his observations (1982 and 1984), which is in agreement with Whitney (1943). Whitney (1943) found that the primary minimum is $0.11 \mathrm{mag}$ deeper than the secondary. The asymmetry noted by Prager (1941) was also confirmed by Whitney's observations (1943). Awadalla (1988) has stated that the depth varies from colour to colour as well, as from time to time. The light curves analyzed by Leung et al. (1985) show large asymmetry in minima and significant discrepancy in the differential eclipse depths.

In spite that the two sets of observations of CerrutiSola \& Scaltriti (1980) and Zhai \& Zhung (1979) are only about two months apart, both sets of light curves show distinct asymmetry at maximum light, an indication that $\mathrm{BB}$ Peg has undergone relatively rapid

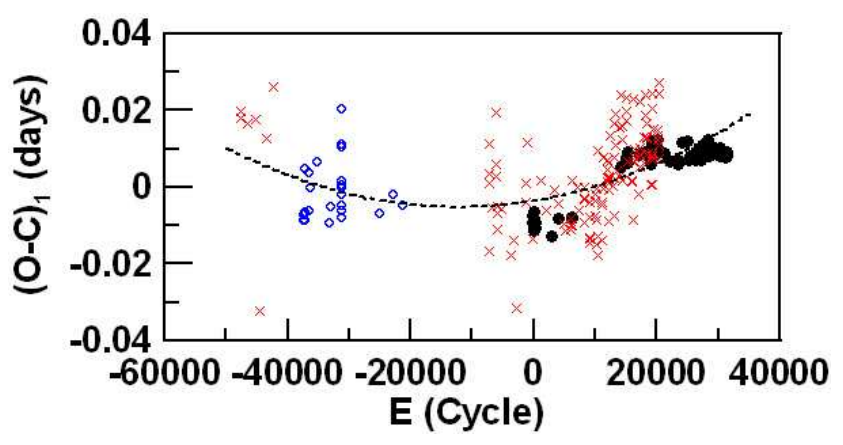

Fig. 4. - The $(O-C)$ diagram of BB Peg constructed with the line elements of Kreiner (2004). The dashed line is the description by a quadratic fit with $S D=0.008$ and $r$ $=0.562$. The open circles, $\mathrm{x}$ signs, and dots, correspond to visual, photographic, and pe \& CCD minima, respectively.

changes (Leung et al. 1985). Giuricin et al. (1981) confirmed that the eclipses are partial, and that the primary minima are occultations.

The asymmetry in the light curves for BB Peg has been modelled with cold spots on the secondary component, the cooler with higher mass and radius (Kalomeni et al. 2007).

In Table 2 we provide the fill out factors, and we show them in Fig. 3. The figure indicates that the size of the envelope varies with the asymmetry of the light curves, and shows an alternative change from HJD 2443750 to 2454359 , i.e. within 29 years. This means that the system has undergone a very rapid variation in this short time.

\section{DATA AND LINE ELEMENTS}

\subsection{Data set}

In order to study the period variation of $\mathrm{BB}$ Peg, the times of the minima (v, pg, pe and CCD) have been carefully collected from the literature (listed in Tables 9 and 10 of the Appendix). Table 9 provides the pe 
and CCD times of the primary and secondary minima, while Table 10 gives the visual (v) and photographic (pg) minima.

\subsection{Line Elements}

Whitney (1943) has determined the $1^{\text {st }}$ true orbital period value of the system BB Peg, after several approximate trials by various authors. However, he has obtained the line elements:

$$
\text { JDHel. }(\text { MinI })=2430285.754+0.3615072 \cdot E .
$$

Later on, several authors have obtained different line elements. In the present study we construct the $O-$ $C$ diagram (Fig. 4) using all the available times of minima data (Tables 9 \& 10), with Kreiner's (2004) line elements:

$$
\text { JDHel. }(\text { MinI })=2443764.3416+0.36150147 \cdot E .
$$

A quadratic least-square fitting of the $O-C$ values has been performed; it yields the following ephemeris:

$$
\begin{aligned}
\text { JDHel. }(\text { MinI })= & 2443764.3381+0.361501737 \cdot E \\
& +1.08 \times 10^{-11} \cdot E^{2},
\end{aligned}
$$

with standard deviation $S D=0.0085$, regression $r=$ 0.5616 , and a rate of increment in the period $d P / d t=$ $2.16 \times 10^{-11} \mathrm{~d} /$ cycle $\left(=2.19 \times 10^{-8} \mathrm{~d} / \mathrm{yr}\right)$ associated with the mass-loss from the secondary to the primary component.

By studying the $O-C$ values from Fig. 4, one should question the accuracy of the results obtained in the previous paragraph. The scatter is very big, especially for visual and photographic observations. If we only consider the pe and CCD minima times (Table 9), which are quite unscattered, we obtain the following new linear and quadratic ephemerides, respectively (Fig. 5):

$$
\text { JDHel. }(\text { MinI })=2443764.33319+0.361502127 \cdot E \text {, }
$$

with $S \mathrm{D}=0.003$ and $r=0.915$;

$$
\begin{aligned}
\text { JDHel. }(\mathrm{MinI})= & 2443764.3383+0.361502946 \cdot \mathrm{E} \\
& -2.78 \times 10^{-11} \cdot E^{2},
\end{aligned}
$$

with $S D=0.002$ and $r=0.963$.

All the line elements of the system given by previous authors, together with the line elements obtained in this work, are listed in Table 3.

\section{PERIOD VARIATION STUDIES}

An essential method to study the period variation in eclipsing binary systems is the analysis of the $O-$ $C$ diagram, by the use of minima times determined throughout all the observational history of the binary.

The orbital period variations of BB Peg have been studied by Cirruti-Sola \& Scaltrity (1980), Zhai \&

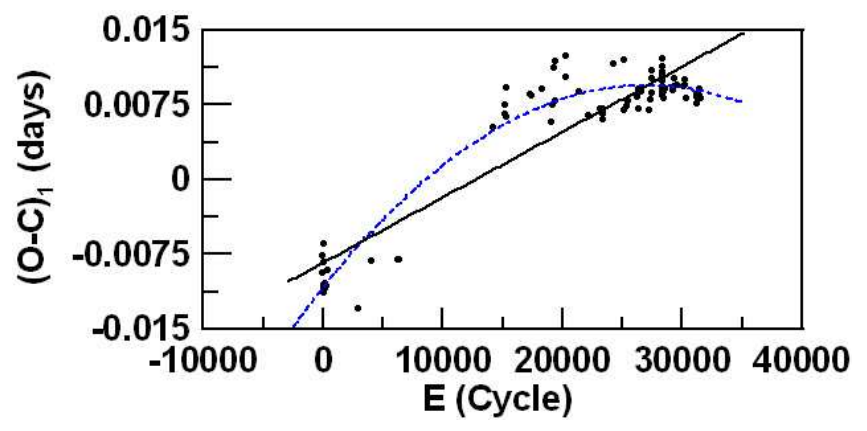

Fig. 5. - Linear and quadratic fits of the $O-C$ residuals for the pe and CCD minima times alone.

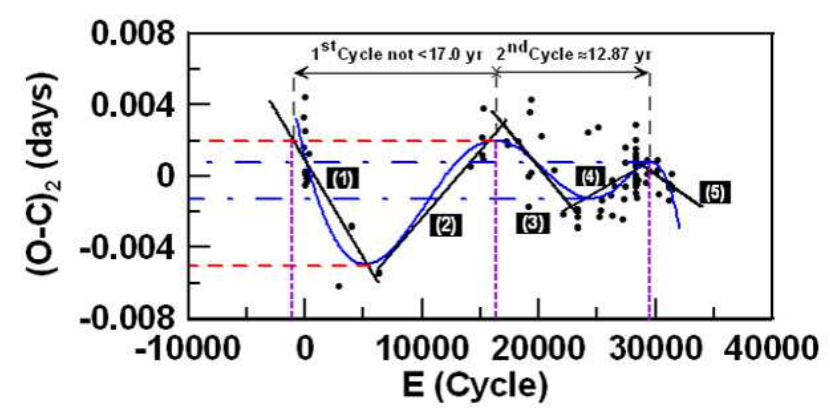

Fig. 6. - Residuals of $\mathrm{BB}$ Peg obtained from the quadratic ephemeris, and their description by several linear ephemerides. The solid curve represents the $7^{\text {th }}$ order polynomial fit with $S D=0.0014$ and $r=0.72$. The vertical and horizontal dashed lines represent the duration and amplitude of each cycle, respectively. (A colour version of this figure is available in the online journal)

Zhang (1977), Awadalla (1988), Qian (2001), Kalomeni et al. (2007) and Snyder (2008). Table 4 provides the comparison between the numbers of data used in previous and present studies.

The general trend of the previous studies suggests: (i) to calculate the changing rate of the orbital period and to refer such change to the mass transfer from the less to the more massive component, (ii) to consider either a hypothetical $3^{\text {rd }}$ companion orbiting the close pair, or the presence of a magnetic activity cycling effect.

\subsection{Mass Transfer}

Awadalla (1988) has collected 43 minimum light times, and pointed out an increase in the orbital period by $\triangle P \simeq 1.5 \times 10^{-7} \mathrm{~d} / \mathrm{yr}$. However, a redetermination of this value gives instead $3.5 \times 10^{-8} \mathrm{~d} / \mathrm{yr}$.

Qian (2001) has used only the pe and CCD minima times in his orbital period study of BB Peg, and reported that the general $O-C$ trend may be increasing continuously. However, he has constructed the $\mathrm{O}-\mathrm{C}$ 


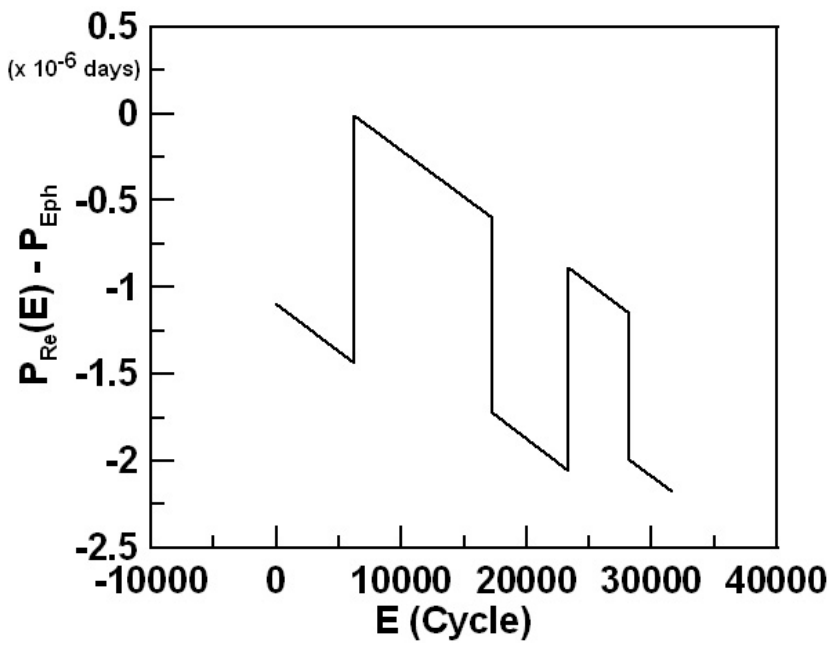

Fig. 7. - Variations in the orbital period of BB Peg. Several jumps in the period are clearly visible.

diagram using 33 ( $\mathrm{v} \& \mathrm{pg}$ ) minima with weight 1 , and 27 (pe \& CCD) minima times with weight 8. Hence, he deduced a continuous period increment rate of 4.75 $\times 10^{-8}$ day $/$ yr.

In the same study, Qian (2001) has found that systems of $\mathrm{W}$-subtype contact binaries showing a period increment usually have higher mass ratios $(q>0.4)$, while periods of low-mass ratio systems $(q<0.4)$ vary in secular decrements. Eq. (5), obtained from the analysis of the $O-C$ diagram of pe and CCD minima times alone, shows a period decrement for BB Peg. Considering the mass ratio $q=0.364$ of $\mathrm{Lu}$ and Rucinski (1999), the obtained secular period decrement agrees with Qian's (2001) result. However, in all the previous studies and in the present analysis of the $O-C$ diagram of BB Peg, one can notice that, when using all the data with high-scattered values (obtained mainly from visual and spectroscopic minima times), the fit produces an orbital period increment rate (see Table $5)$. This increment may be considered a spurious effect.

Kalomeni et al. (2007) have reported a rate of period increment of $3.0 \times 10^{-8} \mathrm{day} / \mathrm{yr}$. They have also reported that this increment can be interpreted in terms of a mass transfer rate of $2.4 \times 10^{-8} \mathrm{M}_{\odot} / \mathrm{yr}$ from the less to the more massive component. Snyder (2008) has deduced a period increase of $2.98 \times 10^{-8}$ day $/ \mathrm{yr}$ instead.

In the present analysis, Eq. 5 shows a decrement rate in the period, $d P / d t=-5.56 \times 10^{-11}$ day $/$ cycle $\left(=-5.62 \times 10^{-8}\right.$ day $\left./ \mathrm{yr}\right)$, associated with mass transfer $(\Delta M)$ from the more to the less massive component.

If the period decrement is caused by conservative mass transfer, one can calculate the mass transfer between the binary components. To estimate the mass transfer rate, the following equation given by Kreiner
\& Ziolkowski (1978) has been used:

$$
\frac{1}{M_{t}} \cdot \frac{d M}{d t}=\frac{q}{3 P\left(q^{2}-1\right)} \cdot \frac{d P}{d t},
$$

where, $M_{t}=M_{1}+M_{2}$ and $q=M_{2} / M_{1}$.

Adopting the values $0.53 \mathrm{M}_{\odot}$ and $1.42 \mathrm{M}_{\odot}$ for $M_{1}$ and $M_{2}$, respectively (Kalomeni et al. 2007), the mass transfer rate is $-4.38 \times 10^{-8} \mathrm{M}_{\odot} / \mathrm{yr}$, from the more massive secondary star to the less massive primary one. This result shows mass transfer from the cooler secondary massive star to the primary less massive one. However, the obtained result is in contradiction with other previous results (see Table 5).

\subsection{Third Body Hypothesis}

D'Angelo et al. (2006) searched for spectroscopic signature of a tertiary by fitting the spectrum of the contact binary BB Peg, and checked if adding a spectrum of a fainter tertiary improves the fit significantly. They motivated their technique in the same study, and detected $M$ dwarf signatures in the spectra. In principle, a direct detection of the third component via spectroscopic observations is preferable in detecting the tertiary, but it comes with some difficulties. These difficulties arise from the complex nature of the W UMa type binaries due to the high rotational velocity of the binary components, the mass transfer and/or loss, and the chromospheric activity which causes high noise in the spectra.

Pribulla and Rusinski (2006) have studied the $O-C$ diagram and reported that the light time effect (LITE) for BB Peg is a marginal detection. However, they have given orbital parameters for a hypothetical third body with $P_{3}=20.4$ yr (Table 6 ).

Irwin's (1959) LITE technique has several advantages over many other approaches. The advantages and disadvantages of the LITE approach have been summarized by Pribulla and Rucinski (2006). However, these authors have reported that: (1) the detection of

Table 5 .

Previous studies and this work

\begin{tabular}{lcc}
\hline \hline & $\begin{array}{c}d P / d t(\mathrm{~d} / \mathrm{yr}) \\
\left(\times 10^{-8}\right)\end{array}$ & $\begin{array}{c}\Delta M_{2}\left(\mathrm{M}_{\odot} / \mathrm{yr}\right) \\
\left(\times 10^{-8}\right)\end{array}$ \\
\hline Cerruti-Sola\& & +4.65 & - \\
Scaltriti(1980) & & \\
Awadalla (1988) & $+3.5^{\dagger}$ & - \\
Qian (2001) & +4.75 & - \\
Kalomeni (2007) & +3.00 & 2.4 \\
Snyder (2008) & +3.00 & - \\
Present work & -5.62 & -4.38 \\
\hline
\end{tabular}

†The new corrected value, instead of the one in the original paper $\left(+15.0 \times 10^{-8} \mathrm{~d} / \mathrm{yr}\right)$. 
Table 6.

Orbital parameters of the hypothetical $3^{\text {rd }}$ body by various authors

\begin{tabular}{lcccc}
\hline \hline & $\begin{array}{c}\text { Pribulla \& } \\
\text { Rucinski }(2006)\end{array}$ & $\begin{array}{c}\text { D'Angelo et al. } \\
(2006)\end{array}$ & $\begin{array}{c}\text { Kalomeni } \\
(2007)\end{array}$ & $\begin{array}{c}\text { Snyder } \\
(2008)\end{array}$ \\
\hline$P_{3}$ (period in yrs.) & $20.4(4)$ & - & $27.9(2)$ & $35.5(1.1)$ \\
$a$ (full amplitude., in days) & 0.0092 & - & - & - \\
$e_{3}$ (eccentricity) & $0.32(21)$ & - & $0.56(0.3)$ & - \\
$\omega_{3}$ long. preias. pass. & - & - & $69(18)$ & - \\
$f\left(M_{3}\right)\left(\mathrm{M}_{\odot}\right)$ & $0.0014(5)$ & - & $0.0010(5)$ & - \\
$M_{3}\left(\mathrm{M}_{\odot}\right)$ & - & 0.51 & - & - \\
$M_{3}\left(\mathrm{M}_{\odot}\right)$ & 0.19 & - & 0.16 & - \\
$M_{3}\left(\mathrm{M}_{\odot}\right)_{i=30}$ & - & - & 1.23 & - \\
$a_{12} \sin i($ projection of & $0.83(9)$ & - & $0.96(15)$ & - \\
semi-major axis, AU) & & & & - \\
no. of $3^{\text {rd }}$ body cycles & 3.59 & - & - & - \\
\hline
\end{tabular}

Table 7.

Four jumps in the orbital period of BB Pegasi

\begin{tabular}{llc}
\hline \hline Interval in cycles & $\Delta T$ (days) & $\Delta P\left(10^{-7}\right.$ days $)$ \\
\hline-96.5 to 6257 & +0.0009 & -0.1100 \\
6257 to 17253 & -0.0033 & +3.3085 \\
17253 to 23345 & +0.0163 & -7.9116 \\
23345 to 28293 & -0.0103 & +3.7620 \\
28293 to 31486 & +0.0140 & -4.6550 \\
\hline
\end{tabular}

short-period orbits may be negatively influenced by the enhanced surface activity; migrating spots can cause wavelike behaviour of the $(O-C)$ diagrams, and only increase the scatter of observed minima times; (2) another possible interpretation of the cyclic period variation is a periodic transfer of orbital angular momentum to magnetic momentum in active systems (Applegate 1992).

A recent study of the $(O-C)$ diagram has been carried out by Snyder (2008), who has determined a third body orbital period value $\left(P_{3}=35.522 \mathrm{yr}\right)$ which is higher than the values obtained by previous authors (see Table 6). However, he has not confirmed nor supported the triplicity of the system by determining any other orbital parameter for the third body. He has considered such high deviation between his and other results given by Kalomeni et al. (2007) or D'Angelo (2006), and argued that the discrepancy is due to the method of analysis. In fact, this is not acceptable for the following reasons: (1) he has used scattered unweighted data in constructing the $O-C$ diagram, while Kalomeni et al. (2007) have given a suitable weight for each type of the observed data; (2) in spite that D'Angelo (2006) and Kalomeni (2007) have determined $P_{3}$ via two different methods, they obtained two comparable values of $P_{3}$ (29.7 and 27.9 years, respectively), which are off from Snyder's (2008) value of 35.522 years.
From the above investigations and because of: (1) the conflict between results obtained from the previous studies for determining $M_{3}$ and $P_{3}$, (2) the observed high scatter in the (v and $\mathrm{pg}$ ) collected data, (3) the chromospheric activity nature of BB Peg, and, (4) the light curve morphology variation, we have aimed in the present $O-C$ study to illustrate the non-continuous variation superimposed on a secular decrement due to mass transfer and/or loss from the system.

Subtracting off the effect of mass transfer or mass loss from the system, we obtain the $(O-C)_{2}$ residual plot in Fig. 6, which shows a significant quasisinusoidal variation. A $7^{\text {th }}$ order polynomial fit with standard deviation $\mathrm{SD}=0.0014$ and correlation coefficient $r=0.7241$ is the solid curve in the figure. In spite of using such a high degree polynomial, the data are not well-fitted. Hence, more details in studying such behavior have to be considered.

The $(O-C)_{2}$ values in Fig. 6 clearly suggest a non-continuous variation. Four clear jumps have taken place in the period of BB Peg within a time interval of 31.18 years which covers two complete cycles. The first cycle is not less than 17.0 years, and the second cycle is of about 12.87 years. Between these jumps, the period is assumed to have undergone a steady decrement. Similar systems, such as TZ Boo and Y Sex, have been studied by Awadalla et al. (2006) and Qian \& Liu (2000). Using the least squares method, a linear func- 
tion in each portion is used to obtain the best fit to the $(O-C)_{2}$ values:

$$
(O-C)_{2}=\Delta T+\Delta P \times E
$$

the values of $\Delta T$ and $\Delta P$ in each portion are listed in Table 7 . The period at any cycle $\mathrm{E}$ has been computed with the following equation:

$$
P_{R e}(E)=P_{E p h}+\Delta P+\frac{d P}{d E} \times E ;
$$

results are shown in Fig. 7, where we have plotted the difference between the real $P_{R e} .(E)$ and the ephemeris $P_{E p h} .\left(0^{d} .36150147\right)$ - in units of $10^{-6}$ day - as a function of time.

An alternative method has been suggested by Hanna (2010). He has divided the variations of the $O-C$ diagram of the system V839 Oph into cycles, in order to highlight its cyclic behaviour. BB Peg may be considered as a similar case to V839 Oph. The $7^{\text {th }}$ order polynomial fit of Fig. 6 shows two cycles, with different durations and different amplitudes.

\section{MAGNETIC ACTIVITY VARIATION}

Applegate (1992) has shown that variations of the subsurface magnetic field which may be compared to the solar activity cycles can lead to a difference between the rotational velocity of the core and that of the outer layer of the convective star. Consequently, the distribution of the angular momentum of this star will vary, and the binary will respond by changing the orbital period. This theory provides a plausible explanation of the observed cyclic period variations of such chromospherically active stars.

As far as magnetic activity, star-spots are expected to be on the cooler, rather than on the hotter star. Hence, the secondary (cool) more massive star has been considered as the active component, when applying the

Table 8.

Magnetic circulation elements from the Applegate mechanism

\begin{tabular}{lll}
\hline \multicolumn{3}{c}{ Applegate mechanism } \\
\hline \hline & $1^{\text {st }}$ Cycle & $2^{n d}$ Cycle \\
$\Delta P / P$ & $3.550 \times 10^{-6}$ & $0.880 \times 10^{-6}$ \\
$\Delta P($ Sec. $)$ & 0.1107 & 0.0276 \\
$\Delta J\left(\mathrm{~g} \mathrm{~cm}^{2} \mathrm{~s}^{-1}\right)$ & $1.490 \times 10^{47}$ & $0.370 \times 10^{47}$ \\
$\Delta \Omega / \Omega$ & $3.250 \times 10^{-4}$ & $0.810 \times 10^{-4}$ \\
$\Delta E(\mathrm{ergs})$ & $2.927 \times 10^{40}$ & $1.815 \times 10^{40}$ \\
$\Delta L_{R M S}\left(\mathrm{ergs} \mathrm{s}^{-1}\right)$ & $1.720 \times 10^{32}$ & $0.140 \times 10^{32}$ \\
$\Delta L_{R M S} / L$ & 0.024 & 0.002 \\
$\mathrm{~B}(\mathrm{kG})($ the mean & 8.50 & 4.88 \\
sub-surface field $)$ & & \\
\hline
\end{tabular}

Applegate's (1992) mechanism. In addition, it is the donor component, as clearly suggested by the quadratic term of Eq. 5 .

Adopting the Applegate's formalism, we consider the two unequal duration cycles found in the $(O-C)_{2}$ diagram to be the modulation periods $P_{\text {mod }}$ : these are $P_{\bmod 1}=17.0$ and $P_{\bmod 2}=12.87$ years, with amplitudes equal to 0.0071 and 0.0013 day, respectively. Moreover, using the parameters given by Kalomeni et al. $(2007)\left(M_{2}=1.42 \mathrm{M}_{\odot}, R_{2}=1.29 \mathrm{R}_{\odot}, L_{2}=\right.$ $1.86 \mathrm{~L}_{\odot}$, and their obtained mean value for the orbital semi-major axes $\left.a=2.667 \mathrm{R}_{\odot}\right)$, and assuming that there is no energy storage in the outer layers of the active cool star, we apply the Applegate's (1992) procedure.

The required value for the angular momentum transfer $\Delta J$ which produces the observed orbital period variations, the energy required to transfer this $\Delta J$, the RMS luminosity variations $\Delta L_{R M S}$, and the magnetic field strength that sustains the whole mechanism, have been computed for both cycles; that are given in Table 8.

The quantities obtained in Table 8 are consistent with and close to those derived by the Applegate's (1992) model, for similar chromospherically active stars.

\section{DISCUSSION AND CONCLUSIONS}

We summarize the asymmetry of the light curves obtained from morphology studies of the binary system BB Peg, as well as its evolutionary status in view of all collected published light curves and the $(O-C)$ diagram, as follows. (1) The photoelectric observations for the system show an occultation primary (Giuricin et al. 1981) or a flat bottom primary (Zola et al. 2005). The occulted duration varies with time over all the light curves of the system. This can be interpreted as a swilling of the bigger star radius, which is due to mass exchange between the binary components. (2) All the light curves of the system show a distinct O'Connell effect, which means that the system has magnetic activity due to a group of cool spots. These spots may be found on one or on both components with cycling of about 23 years (see Table 1 and Fig. 1). (3) The change in depths refers to the luminosity variations of the components, that may confirm the presence of the cool spots on the system. (4) The different fill-out ratio values (Table 2 \& Fig. 3) obtained by previous authors may be due to changes in the asymmetric structure; these changes are usually caused by surface brightness anomalies due to mass exchange between the binary components. The short-time variation of the light curves for the system could be explained by active envelope and/or mass exchange, as well as by magnetic activity cycling. However, the chromospheric and coronal activities on W UMa binaries are common and have been taken into consideration in the liter- 
ature. (5) A careful study of the $(O-C)$ diagram shows that the cause of the quasi-periodic variation is not due to the LITE as a result of the presence of a third body; however, some authors have determined the elements of third body and have tried to prove its presence spectroscopically (D'Angelo et al. 2006) by various techniques.

A possible explanation is magnetic activity due to the presence of star spots, which affects the period variation cyclically- as it is common in chromospheric active late type stars. Such cyclic magnetic activity variation (not strictly periodic) is superimposed to the mass exchange between the binary components with jumps of its orbital period behaviour (Fig. 7).

The present study shows mass transfer from the more to the less massive component, which contradicts previous studies. The difference between our result and previous studies may be due to the use of all the available high quality data, represented in terms of the pe and CCD minima times.

Notice also that when we used pg and v data together with pe and CCD minima (as previous authors), we obtained an increment in orbital period rate which is similar to their results.

The analysis of the $(\mathrm{O}-\mathrm{C})$ diagram shows variations in the period, which can be explained either by stellar magnetic activity cycles on the cool secondary more massive component (with subsurface magnetic fields equal to $8.5 \mathrm{kG}$ and $4.88 \mathrm{kG}$ for the two cycles), or by a third companion orbiting the close pair. These magnetic activity cycling may be superimposed on a long term orbital period modulation decrement of rate $d P / d t=-5.62 \times 10^{-8} \mathrm{~d} / \mathrm{yr}$, corresponding to a timescale of $6.43 \times 10^{6}$ years.

Further accurate photoelectric and CCD observations are required to determine any asymmetry for the binary system BB Peg, as well as to confirm the magnetic activity cycling to be the cause of such quasisinusoidal variation in the $O-C$ diagram.

\section{ACKNOWLEDGMENTS}

The authors would like to thank the anonymous referee for his comments and efforts in modifying the text. This paper has made use of the variable star observations from the AAVSO and the BBSAG International Database contributed by observers worldwide. Many thanks to the NASA Astrophysics Data Service and to the Cracow Eclipsing Binaries Minima Database.

\section{REFERENCES}

Applegate, J. H. 1992, A Mechanism for Orbital Period Modulation in Close Binaries, ApJ, 385, 621

Awadalla, N. S. 1988, Three-Colours Photoelectric Observations of W UMa-System BB Pegasi, Ap\&SS, 140, 137

Awadalla, N. S., Hanna, M. A., Saad, A. S., \& Morcos, A. B. 2006, Study of Magnetic Activity and Period Variations of TZ Bootis System, CoSka, 36, 47

Cerruti-Sola, M., \& Scaltriti, F. 1980, Two-Color Photoelectric Observations of the Eclipsing Binary BB Peg, A\&AS, 40, 85

Cerruti-Sola, M., Milano, L., \& Scaltriti, F. 1981, BB Pegasi: A W UMa-W System with a High Degree of Overcontact, A\&A, 101, 273

Giuricin, G., Mardirossian, F., \& Mezzetti, M. 1981, Light Curve Synthesis for the Eclipsing Binary BB Peg, AN, 302, 285

D’Angelo, C., Van Kerkwijk, M. H., \& Rucinski, S. M. 2006, Contact Binaries with Additional Components. II. A Spectroscopic Search for Faint Tertiaries, AJ, 132, 650

Hanna, M. A. 2010, Period Variation Study of the A-Type W UMa Eclipsing Binary V839 Oph, JKAS, 43, 201

Hoffmeister, C. 1931, 316, neue Vernderliche, AN, 242, 129

Hrivnak, B. J. 1990, Radial Velocity Studies of the W UMa Binaries BX Peg and BB Peg, BAAS, 22, 1291

Irwin, J. B. 1959, Standard Light-Time Curves, AJ, 64, 149

Kalomeni, B., Yakut, K., \& Keskin, V. 2007, Absolute Properties of the Binary System BB Pegasi, AJ, 134, 642

Kreiner, J. M., \& Ziolkowski, J. 1978, Period Changes and Evolutionary Status of 18 Algol-Type Systems, AcA, 28, 497

Kreiner, J. M. 2004, Up-To-Date Linear Elements of Eclipsing Binaries, AcA, 54, 207

Leung, K. C., Zhai, D., \& Zhang, Y. 1985, Two very Similar Late-Type Contact Systems - BX And and BB Pegasi, AJ, 90, 515

Lu, W., \& Rucinski, S. M. 1999, Radial Velocity Studies of Close Binary Stars. I., AJ, 118, 515

Prager, R. 1941, History and Bibliography of the Light Variations of Variable Stars, AnHar, 111, 1

Pribulla, T., \& Rucinski, S. 2006, Contact Binaries with Additional Components. I. The Extant Data, AJ, 131, 2986

Qian, S., \& Liu, Q. 2000, A Possible Connection between the Variation of Light Curve and the Change of the Orbital Period in the Contact Binary CK Bootis, Ap\&SS, 271, 331

Qian, S. 2001, A Possible Relation between the Period Change and the Mass Ratio for W-Type Contact Binaries, MNRAS, 328, 635

Russell, H. N., \& Merrill, J. E. 1952, The Determination of the Elements of Eclipsing Binaries, Contr. Princeton Univ. No. 26

Synder, L. F. 2008, Eclipsing Binary System BB Pegasus, SASS, 27, 153

Wilson, R. E., \& Devinney, E. J. 1971, Realization of Accurate Close Binary Light Curves: Application to MR Cygni, ApJ, 166, 605

Whitney, B. S. 1943, Some Eclipsing Variable Stars, AJ, 50, 131

Whitney, B. S. 1959, Minima and Periods of Eclipsing Stars, AJ, 64, 258 
Wood, D. B. 1972, A Computer Program for Modelling Non-Spherical Eclipsing Binary System, Gorddard Space Flight Center Greenbelt, USA

Zhai, D. S., \& Zhang, R. X. 1979, Photoelectric Observations of Two Totally Eclipsing W Ursae Majoris Systems: BB Peg and BX Peg., Kexue Tongbao, 24, 986

Zola, S., Kreiner, J. M., \& Zakrzewski, B. et al. 2005, Physical Parameters of Components in Close Binary Systems $\mathrm{V}, \mathrm{AcA}, 55,389$

\section{APPENDIX A. TIMES OF MINIMA}

Table 9.

Photoelectric and CCD minima times

\begin{tabular}{|c|c|c|c|c|}
\hline $\begin{array}{l}\text { HJD. } \\
(+2400000)\end{array}$ & $\bar{E}$ & $(O-C)$ & Type & Ref. \\
\hline 43729.4491 & -96.5 & -0.00761 & pe & 1 \\
\hline 43730.3512 & -94 & -0.00926 & pe & {$[1]$} \\
\hline 43750.5940 & -38 & -0.01054 & pe & {$[2]$} \\
\hline 43754.3890 & -27.5 & -0.01131 & pe & [2] \\
\hline 43754.3896 & -27.5 & -0.01071 & pe & [1] \\
\hline 43757.4667 & -19 & -0.00637 & pe & {$[1]$} \\
\hline 43764.3334 & 00.0 & -0.00820 & pe & {$[1]$} \\
\hline 43806.0845 & 115.5 & -0.01049 & pe & [3] \\
\hline 43806.9884 & 118 & -0.01039 & pe & [3] \\
\hline 43813.1337 & 135 & -0.01065 & pe & [3] \\
\hline 43814.0371 & 137.5 & -0.01095 & pe & [3] \\
\hline 43842.0537 & 215 & -0.01069 & pe & [3] \\
\hline 43866.9989 & 284 & -0.00909 & pe & [3] \\
\hline 44812.5022 & 2899.5 & -0.01291 & pe & {$[4]$} \\
\hline 45208.3511 & 3994.5 & -0.00812 & pe & {$[5]$} \\
\hline 45208.5319 & 3995 & -0.00807 & pe & {$[5]$} \\
\hline 46024.2600 & 6251.5 & -0.00804 & pe & [5] \\
\hline 46026.2483 & 6257 & -0.00800 & pe & {$[5]$} \\
\hline 48887.3650 & 14171.5 & 0.00532 & ccd & {$[6]$} \\
\hline 49243.4462 & 15156.5 & 0.00757 & pe & {$[7]$} \\
\hline 49244.3490 & 15159 & 0.00662 & pe & {$[7]$} \\
\hline 49273.2689 & 15239 & 0.00640 & pe & {$[7]$} \\
\hline 49275.2600 & 15244.5 & 0.00924 & pe & {$[7]$} \\
\hline 50001.3351 & 17253 & 0.00864 & pe & [8] \\
\hline 50026.2785 & 17322 & 0.00844 & pe & [8] \\
\hline 50359.4028 & 18243.5 & 0.00913 & ccd & [9] \\
\hline 50657.4575 & 19068 & 0.00587 & pe & {$[10]$} \\
\hline 50671.3770 & 19106.5 & 0.00756 & pe & [10] \\
\hline 50702.4698 & 19192.5 & 0.01124 & ccd & [11] \\
\hline 50739.7052 & 19295.5 & 0.01199 & $\operatorname{ccd}$ & {$[12]$} \\
\hline 50769.5250 & 19378 & 0.00791 & $\operatorname{ccd}$ & {$[12]$} \\
\hline 51078.4304 & 20232.5 & 0.01031 & ccd & {$[13]$} \\
\hline 51471.3810 & 21319.5 & 0.00881 & $\operatorname{ccd}$ & {$[14]$} \\
\hline 51770.5212 & 22147 & 0.00654 & pe & [15] \\
\hline 52131.8425 & 23146.5 & 0.00712 & ccd & {$[16]$} \\
\hline 52201.2508 & 23338.5 & 0.00714 & pe & {$[17]$} \\
\hline 52201.4305 & 23339 & 0.00609 & pe & {$[17]$} \\
\hline 52203.2386 & 23344 & 0.00668 & pe & {$[17]$} \\
\hline 52203.4188 & 23344.5 & 0.00613 & pe & {$[17]$} \\
\hline 52207.3962 & 23355.5 & 0.00702 & pe & {$[17]$} \\
\hline 52513.4118 & 24202 & 0.01162 & ccd & [18] \\
\hline 52838.4020 & 25101 & 0.01200 & ccd & [19] \\
\hline 52852.4956 & 25140 & 0.00704 & pe & {$[20]$} \\
\hline 52903.4676 & 25281 & 0.00738 & ccd & {$[21]$} \\
\hline
\end{tabular}

Table 9. - continued

\begin{tabular}{lllll}
\multicolumn{5}{c}{ Table 9. - continued } \\
\hline HJD & E & $(O-C)$ & Type & Ref. \\
$(+2400000)$ & & & & \\
\hline 52956.2474 & 25427 & 0.00795 & ccd & {$[21]$} \\
53243.4607 & 26221.5 & 0.00830 & ccd & {$[22]$} \\
53266.4149 & 26285 & 0.00717 & ccd & {$[21]$} \\
53284.3112 & 26334.5 & 0.00914 & pe & {$[17]$} \\
53285.3957 & 26337.5 & 0.00913 & pe & {$[17]$} \\
53353.3577 & 26525.5 & 0.00886 & ccd & {$[22]$} \\
53591.4046 & 27184 & 0.00703 & ccd & {$[21]$} \\
53638.4009 & 27314 & 0.00814 & ccd & {$[21]$} \\
53661.3569 & 27377.5 & 0.00881 & pe & {$[23]$} \\
53661.5390 & 27378 & 0.01015 & pe & {$[23]$} \\
53675.2769 & 27416 & 0.01100 & pe & {$[23]$} \\
53684.3136 & 27441 & 0.01020 & ccd & {$[21]$} \\
53984.3589 & 28271 & 0.00924 & ccd & {$[22]$} \\
53984.3591 & 28271 & 0.00944 & ccd & {$[22]$} \\
53984.5409 & 28271.5 & 0.01049 & ccd & {$[22]$} \\
53984.5411 & 28271.5 & 0.01069 & ccd & {$[22]$} \\
53986.3485 & 28276.5 & 0.01058 & pe & {$[24]$} \\
53986.5271 & 28277 & 0.00843 & ccd & {$[22]$} \\
53986.5276 & 28277 & 0.00893 & ccd & {$[22]$} \\
53987.4330 & 28279.5 & 0.01058 & pe & {$[24]$} \\
53988.3352 & 28282 & 0.00903 & pe & {$[25]$} \\
53988.5175 & 28282.5 & 0.01057 & pe & {$[26]$} \\
53990.3248 & 28287.5 & 0.01037 & pe & {$[27]$} \\
53990.5040 & 28288 & 0.00882 & pe & {$[28]$} \\
53991.4089 & 28290.5 & 0.00996 & ccd & {$[29]$} \\
53992.4949 & 28293.5 & 0.01146 & ccd & {$[22]$} \\
53992.4957 & 28293.5 & 0.01226 & ccd & {$[22]$} \\
54008.5804 & 28338 & 0.01014 & ccd & {$[30]$} \\
54009.3042 & 28340 & 0.01091 & ccd & {$[21]$} \\
54037.3178 & 28417.5 & 0.00818 & pe & {$[24]$} \\
54039.3068 & 28423 & 0.00892 & ccd & {$[31]$} \\
54086.3022 & 28553 & 0.00913 & ccd & {$[32]$} \\
54298.5034 & 29140 & 0.00896 & ccd & {$[33]$} \\
54360.3206 & 29311 & 0.00941 & pe & {$[34]$} \\
54366.6477 & 29328.5 & 0.01024 & ccd & {$[35]$} \\
54444.5506 & 29544 & 0.00957 & ccd & {$[36]$} \\
54631.8082 & 30062 & 0.00941 & ccd & {$[37]$} \\
54658.7402 & 30136.5 & 0.00955 & ccd & {$[37]$} \\
54678.8041 & 30192 & 0.01012 & ccd & {$[37]$} \\
54710.6143 & 30280 & 0.00819 & ccd & {$[37]$} \\
55022.7710 & 31143.5 & 0.00837 & ccd & {$[38]$} \\
55033.4355 & 31173 & 0.00858 & ccd & {$[33]$} \\
55044.8219 & 31204.5 & 0.00768 & ccd & {$[36]$} \\
55089.4688 & 31328 & 0.00915 & ccd & {$[36]$} \\
55096.6981 & 31348 & 0.00842 & ccd & {$[36]$} \\
55146.5851 & 31486 & 0.00822 & ccd & {$[36]$} \\
\hline & & & &
\end{tabular}

Ref: [1] A\&AS, 40, 85., [2] IBVS 2023., [3] Zhai\&Zhang, 1979 (cf., AJ. 143, 647), [4] IBVS 2159., [5] Awadalla, 1988, Ap\&SS, 140,137. [6] BBSAG Bull., 102., [7] IBVS 4380., [8] IBVS 4382., [9] IBVS 4562., [10] IBVS 4534., [11] IBVS 4606., [12] AAVSO 5., [13] IBVS 4712., [14] IBVS 5017., [15] IBVS 5296., [16] IBVS 5224., [17] IBVS 5623., [18] IBVS 5364., [19] IBVS 5464., [20] IBVS 5643., [21] B.R.N.O. Contrib. 34, 2007. [22] Kalomeni et al., 2007, AJ, 143, 647., [23] IBVS 5731., [24] IBVS 5736., [25] IBVS 5737., [26] IBVS 5738., [27] IBVS 5739., [28] IBVS 5740., [29] IBVS 5746., [30] IBVS 5843., [31] IBVS 5777., [32] IBVS 5897., [33] IBVS 5898., [34] IBVS 8530., [35] JAAVSO, 2008, 36, 171., [36] IBVS 5814., [37] JAAVSO, 2008, 36, 186., [38] JAAVSO, 2010, 38, 1 . 
Table 10.

Visual and photographic minima times

\begin{tabular}{|c|c|c|c|c|}
\hline $\begin{array}{l}\text { HJD } \\
(+2400000)\end{array}$ & $\overline{\mathrm{E}}$ & $(O-C)_{1}$ & Type & Ref. \\
\hline 26559.241 & -47593.5 & 0.01961 & $\mathrm{~V}$ & [1] \\
\hline 26582.014 & -47530.5 & 0.01802 & $\mathrm{v}$ & [2] \\
\hline 26965.204 & -46470.5 & 0.01646 & $\mathrm{~V}$ & [2] \\
\hline 27393.223 & -45286.5 & 0.01772 & $\mathrm{~V}$ & [2] \\
\hline 27675.144 & -44506.5 & -0.03243 & $\mathrm{~V}$ & [3] \\
\hline 28058.561 & -43446 & 0.01267 & $\mathrm{~V}$ & [4] \\
\hline 28498.522 & -42229 & 0.02598 & $\mathrm{v}$ & [5] \\
\hline 30226.826 & -37448 & -0.00855 & pg & {$[6]$} \\
\hline 30235.865 & -37423 & -0.00709 & pg & [7] \\
\hline 30258.638 & -37360 & -0.00868 & pg & [7] \\
\hline 30264.797 & -37343 & 0.00479 & $\mathrm{pg}$ & [7] \\
\hline 30281.776 & -37296 & -0.00677 & $\mathrm{pg}$ & [7] \\
\hline 30285.753 & -37285 & -0.00629 & $\mathrm{pg}$ & [7] \\
\hline 30530.861 & -36607 & 0.00371 & $\mathrm{pg}$ & [7] \\
\hline 30552.903 & -36546 & -0.00588 & $\mathrm{pg}$ & {$[7]$} \\
\hline 30584.721 & -36458 & -0.00001 & pg & {$[7]$} \\
\hline 30994.128 & -35325.5 & 0.00658 & pg & [2] \\
\hline 31731.756 & -33285 & -0.00917 & $\mathrm{pg}$ & [8] \\
\hline 31783.455 & -33142 & -0.00488 & $\mathrm{pg}$ & [8] \\
\hline 32433.631 & -31343.5 & 0.01072 & $\mathrm{pg}$ & [8] \\
\hline 32433.801 & -31343 & -0.00003 & $\mathrm{pg}$ & [8] \\
\hline 32436.866 & -31334.5 & -0.00779 & pg & [8] \\
\hline 32436.687 & -31335 & -0.00604 & $\mathrm{pg}$ & [8] \\
\hline 32451.697 & -31293.5 & 0.00165 & $\mathrm{pg}$ & [8] \\
\hline 32455.683 & -31282.5 & 0.01114 & $\mathrm{pg}$ & [8] \\
\hline 32473.567 & -31233 & 0.00081 & $\mathrm{pg}$ & [8] \\
\hline 32477.538 & -31222 & -0.00470 & pg & [8] \\
\hline 32477.744 & -31221.5 & 0.02055 & $\mathrm{pg}$ & [8] \\
\hline 32479.710 & -31216 & -0.00171 & $\mathrm{pg}$ & [8] \\
\hline 34711.615 & -25042 & -0.00679 & $\mathrm{pg}$ & [8] \\
\hline 35468.604 & -22948 & -0.00187 & $\mathrm{pg}$ & [8] \\
\hline 36056.764 & -21321 & -0.00476 & $\mathrm{pg}$ & [8] \\
\hline 41148.518 & -7236 & 0.00104 & - & $\dagger$ \\
\hline 41176.356 & -7159 & 0.00342 & - & $\dagger$ \\
\hline 41178.352 & -7153.5 & 0.01117 & - & $\dagger$ \\
\hline 41181.397 & -7145 & -0.01660 & $\mathrm{v}$ & [9] \\
\hline 41335.227 & -6719.5 & -0.00547 & $\mathrm{~V}$ & [1] \\
\hline 41561.371 & -6094 & 0.01936 & $\mathrm{~V}$ & [11] \\
\hline 41562.439 & -6091 & 0.00285 & $\mathrm{~V}$ & [11] \\
\hline 41563.346 & -6088.5 & 0.00610 & $\mathrm{~V}$ & [11] \\
\hline 41616.289 & -5942 & -0.01087 & $\mathrm{v}$ & {$[12]$} \\
\hline 41618.283 & -5936.5 & -0.00512 & $\mathrm{v}$ & {$[12]$} \\
\hline 41622.258 & -5925.5 & -0.00664 & $\mathrm{v}$ & [12] \\
\hline 41624.248 & -5920 & -0.00490 & $\mathrm{v}$ & [12] \\
\hline 41863.561 & -5258 & -0.00587 & $\mathrm{v}$ & [13] \\
\hline 42405.259 & -3759.5 & -0.01782 & $\mathrm{v}$ & [14] \\
\hline 42607.523 & -3200 & -0.01390 & $\mathrm{v}$ & {$[15]$} \\
\hline 42748.310 & -2810.5 & -0.03172 & $\mathrm{v}$ & [16] \\
\hline 43337.766 & -1180 & -0.00387 & $\mathrm{~V}$ & [17] \\
\hline 43337.771 & -1180 & 0.00113 & $\mathrm{~V}$ & [17] \\
\hline 43346.819 & -1155 & 0.01160 & $\mathrm{~V}$ & [17] \\
\hline 43705.765 & -162 & -0.01336 & $\mathrm{~V}$ & [17] \\
\hline 44191.638 & 1182 & 0.00166 & $\mathrm{v}$ & [17] \\
\hline 44474.686 & 1965 & -0.00599 & $\mathrm{v}$ & [17] \\
\hline 44944.643 & 3265 & -0.00090 & $\mathrm{~V}$ & [17] \\
\hline 45193.714 & 3954 & -0.00441 & $\mathrm{~V}$ & [17] \\
\hline 45578.708 & 5019 & -0.00948 & $\mathrm{~V}$ & [17] \\
\hline 45591.720 & 5055 & -0.01153 & $\mathrm{~V}$ & {$[17]$} \\
\hline 45959.730 & 6073 & -0.01003 & $\mathrm{v}$ & {$[17]$} \\
\hline
\end{tabular}

Table 10. - continued

\begin{tabular}{|c|c|c|c|c|}
\hline $\begin{array}{l}\text { HJD } \\
(+2400000)\end{array}$ & $\bar{E}$ & $(O-C)$ & Type & Ref. \\
\hline 45959.732 & 6073 & -0.00803 & $\mathrm{~V}$ & 17] \\
\hline 45962.621 & 6081 & -0.01104 & $\mathrm{v}$ & {$[17]$} \\
\hline 46017.582 & 6233 & 0.00174 & $\mathrm{v}$ & {$[17]$} \\
\hline 46026.608 & 6258 & -0.00980 & $\mathrm{v}$ & {$[17]$} \\
\hline 46321.603 & 7074 & 0.00000 & $\mathrm{v}$ & {$[17]$} \\
\hline 46413.599 & 7328.5 & -0.00612 & $\mathrm{v}$ & {$[17]$} \\
\hline 46706.418 & 8138.5 & -0.00331 & $\mathrm{v}$ & [18] \\
\hline 46713.642 & 8158.5 & -0.00934 & $\mathrm{v}$ & [17] \\
\hline 46731.365 & 8207.5 & 0.00008 & $\mathrm{v}$ & [19] \\
\hline 46759.559 & 8285.5 & -0.00303 & $\mathrm{v}$ & [17] \\
\hline 47027.420 & 9026.5 & -0.01462 & $\mathrm{v}$ & [20] \\
\hline 47052.365 & 9095.5 & -0.01322 & $\mathrm{v}$ & [20] \\
\hline 47054.353 & 9101 & -0.01348 & $\mathrm{v}$ & [20] \\
\hline 47055.443 & 9104 & -0.00798 & $\mathrm{v}$ & {$[20]$} \\
\hline 47062.677 & 9124 & -0.00401 & $\mathrm{v}$ & {$[17]$} \\
\hline 47391.463 & 10033.5 & -0.00360 & $\mathrm{v}$ & {$[21]$} \\
\hline 47407.365 & 10077.5 & -0.00766 & $\mathrm{v}$ & {$[21]$} \\
\hline 47412.432 & 10091.5 & -0.00168 & $\mathrm{v}$ & [21] \\
\hline 47439.357 & 10166 & -0.00854 & $\mathrm{v}$ & {$[22]$} \\
\hline 47466.297 & 10240.5 & -0.00040 & $\mathrm{v}$ & {$[22]$} \\
\hline 47470.259 & 10251.5 & -0.01492 & $\mathrm{v}$ & {$[22]$} \\
\hline 47524.328 & 10401 & 0.00961 & $\mathrm{v}$ & [22] \\
\hline 47526.294 & 10406.5 & -0.01265 & $\mathrm{v}$ & {$[22]$} \\
\hline 47528.277 & 10412 & -0.01791 & $\mathrm{v}$ & {$[23]$} \\
\hline 47742.480 & 11004.5 & -0.00453 & $\mathrm{v}$ & {$[24]$} \\
\hline 47778.450 & 11104 & -0.00392 & $\mathrm{v}$ & {$[24]$} \\
\hline 47804.468 & 11176 & -0.01403 & $\mathrm{v}$ & {$[25]$} \\
\hline 47857.261 & 11322 & -0.00024 & $\mathrm{v}$ & {$[25]$} \\
\hline 47861.607 & 11334 & 0.00774 & $\mathrm{v}$ & {$[17]$} \\
\hline 47862.330 & 11336 & 0.00774 & $\mathrm{v}$ & [25] \\
\hline 48092.425 & 11972.5 & 0.00705 & $\mathrm{v}$ & {$[26]$} \\
\hline 48114.471 & 12033.5 & 0.00146 & $\mathrm{v}$ & {$[26]$} \\
\hline 48115.378 & 12036 & 0.00471 & $\mathrm{v}$ & {$[26]$} \\
\hline 48162.371 & 12166 & 0.00252 & $\mathrm{v}$ & {$[26]$} \\
\hline 48170.328 & 12188 & 0.00648 & $\mathrm{v}$ & {$[26]$} \\
\hline 48176.639 & 12205.5 & -0.00879 & $\mathrm{v}$ & {$[17]$} \\
\hline 48187.314 & 12235 & 0.00191 & $\mathrm{v}$ & {$[26]$} \\
\hline 48205.390 & 12285 & 0.00284 & $\mathrm{v}$ & [27] \\
\hline 48210.628 & 12299.5 & -0.00093 & $\mathrm{v}$ & {$[17]$} \\
\hline 48219.680 & 12324.5 & 0.01353 & $\mathrm{v}$ & {$[17]$} \\
\hline 48441.448 & 12938 & 0.00038 & $\mathrm{v}$ & [28] \\
\hline 48479.422 & 13043 & 0.01673 & $\mathrm{v}$ & [28] \\
\hline 48500.365 & 13101 & -0.00736 & $\mathrm{v}$ & [28] \\
\hline 48519.359 & 13153.5 & 0.00781 & $\mathrm{v}$ & {$[28]$} \\
\hline 48537.609 & 13204 & 0.00199 & $\mathrm{v}$ & {$[17]$} \\
\hline 48546.302 & 13228 & 0.01895 & $\mathrm{v}$ & [29] \\
\hline 48564.369 & 13278 & 0.01088 & $\mathrm{v}$ & {$[29]$} \\
\hline 48623.287 & 13441 & 0.00414 & $\mathrm{v}$ & {$[30]$} \\
\hline 48837.488 & 14033.5 & 0.01552 & $\mathrm{v}$ & [31] \\
\hline 48843.440 & 14050 & 0.00275 & $\mathrm{v}$ & [31] \\
\hline 48885.757 & 14167 & 0.02407 & $\mathrm{v}$ & {$[17]$} \\
\hline 48936.345 & 14307 & 0.00187 & $\mathrm{v}$ & [31] \\
\hline 48954.615 & 14357.5 & 0.01604 & $\mathrm{v}$ & [17] \\
\hline 49001.245 & 14486.5 & 0.01235 & $\mathrm{v}$ & {$[32]$} \\
\hline 49198.449 & 15032 & 0.01730 & $\mathrm{v}$ & $33]$ \\
\hline 49219.422 & 15090 & 0.02322 & $\mathrm{v}$ & {$[34]$} \\
\hline 49241.651 & 15151.5 & 0.01988 & $\mathrm{v}$ & {$[17]$} \\
\hline 49264.593 & 15215 & 0.00653 & $\mathrm{v}$ & {$[17]$} \\
\hline 49549.451 & 16003 & 0.00138 & $\mathrm{v}$ & [35] \\
\hline
\end{tabular}


Table 10. - continued

\begin{tabular}{llrll}
\hline $\begin{array}{l}\text { HJD } \\
(+2400000)\end{array}$ & $\mathrm{E}$ & $(O-C)$ & Type & Ref. \\
\hline 49561.381 & 16036 & 0.00183 & $\mathrm{v}$ & {$[35]$} \\
49602.597 & 16150 & 0.00666 & $\mathrm{v}$ & {$[17]$} \\
49605.474 & 16158 & -0.00835 & $\mathrm{v}$ & {$[38]$} \\
49633.341 & 16235 & 0.02303 & $\mathrm{v}$ & {$[36]$} \\
49679.598 & 16363 & 0.00785 & $\mathrm{v}$ & {$[17]$} \\
49713.581 & 16457 & 0.00971 & $\mathrm{v}$ & {$[17]$} \\
49917.466 & 17021 & 0.00788 & $\mathrm{v}$ & {$[37]$} \\
49934.447 & 17068 & -0.00169 & $\mathrm{v}$ & {$[37]$} \\
49983.623 & 17204 & 0.01011 & $\mathrm{v}$ & {$[38]$} \\
49989.600 & 17220.5 & 0.02234 & $\mathrm{v}$ & {$[38]$} \\
50002.597 & 17256.5 & 0.00528 & $\mathrm{v}$ & {$[38]$} \\
50014.349 & 17289 & 0.00849 & $\mathrm{v}$ & {$[37]$} \\
50276.442 & 18014 & 0.01292 & $\mathrm{v}$ & {$[39]$} \\
50331.396 & 18166 & 0.01870 & $\mathrm{v}$ & {$[40]$} \\
50357.610 & 18238.5 & 0.02384 & $\mathrm{v}$ & {$[38]$} \\
50370.613 & 18274.5 & 0.01279 & $\mathrm{v}$ & {$[38]$} \\
50376.752 & 18291.5 & 0.00626 & $\mathrm{v}$ & {$[38]$} \\
50390.315 & 18329 & 0.01296 & $\mathrm{v}$ & {$[41]$} \\
50391.584 & 18332.5 & 0.01670 & $\mathrm{v}$ & {$[38]$} \\
50425.557 & 18426.5 & 0.00856 & $\mathrm{v}$ & {$[38]$} \\
50455.562 & 18509.5 & 0.00894 & $\mathrm{v}$ & {$[38]$} \\
50682.215 & 19136.5 & 0.00052 & $\mathrm{v}$ & {$[42]$} \\
50692.705 & 19165.5 & 0.00698 & $\mathrm{v}$ & {$[38]$} \\
50698.671 & 19182 & 0.00820 & $\mathrm{v}$ & {$[38]$} \\
50703.363 & 19195 & 0.00068 & $\mathrm{v}$ & {$[43]$} \\
50726.688 & 19259.5 & 0.00884 & $\mathrm{v}$ & {$[38]$} \\
50731.580 & 19273 & 0.02057 & $\mathrm{v}$ & {$[38]$} \\
50753.274 & 19333 & 0.02448 & $\mathrm{v}$ & {$[43]$} \\
50799.537 & 19461 & 0.01529 & $\mathrm{v}$ & {$[38]$} \\
51012.821 & 20051 & 0.01343 & $\mathrm{v}$ & {$[38]$} \\
51020.773 & 20073 & 0.01239 & $\mathrm{v}$ & {$[38]$} \\
\hline & & & &
\end{tabular}

Table 10. - continued

\begin{tabular}{lllll}
\hline $\begin{array}{l}\text { HJD } \\
(+2400000)\end{array}$ & $\mathrm{E}$ & $(O-C)$ & Type & Ref. \\
\hline 51069.759 & 20208.5 & 0.01494 & $\mathrm{v}$ & {$[38]$} \\
51084.590 & 20249.5 & 0.02438 & $\mathrm{v}$ & {$[38]$} \\
51097.607 & 20285.5 & 0.02733 & $\mathrm{v}$ & {$[38]$} \\
51099.579 & 20291 & 0.01107 & $\mathrm{v}$ & {$[38]$} \\
51129.580 & 20374 & 0.00745 & $\mathrm{v}$ & {$[38]$} \\
\hline
\end{tabular}

$\dagger$ Visual minima times listed in the Crakow Eclipsing Binaries Minima Database, but not presented in their source (BBSAG Bull. 31).

Ref.: [1] Zessewitsch, 1939, Quoted in Princeton Contributions No. 19,.[2] Tsessevich, V. P., 1954, Odessa Izv., 4, 271., [3] Dobronravin, 1935, N.N.V.S., 4, 415., [4] Piotrowski, S., 1936, ibid, 2, 157 [cf., AcA, 1977, 27,151]., [5] Dworak, T.Z., 1977, AcA, 27,151., [6] Whitney, B. S., 1943, AJ, 50, 131., [7] Whitney, B.S., 1959, AJ, 64, 258., [8] Diethelm, R., 1973, Rocznik Astron. Obser. Krakowskiego, 44, 102., [9] BBSAG Bull. 1., [10] BBSAG Bull. 5., [11] BBSAG Bull. 6., [12] BBSAG Bull. 10., [13] BBSAG Bull. 19., [14] BBSAG Bull. 23. [15] BBSAG Bull. 25., [16] AAVSO 2., [17] BBSAG Bull. 81., [18] BBSAG Bull. 83., [19] BBSAG Bull. 86., [20] BBSAG Bull. 89., [21] BBSAG Bull. 90., [22] BBSAG Bull. 91, [23] BBSAG Bull. 92. [24] BBSAG Bull. 93., [25] BBSAG Bull. 96., [26] BBSAG Bull. 97., [27] BBSAG Bull. 98., [28] BBSAG Bull. 99., [29] BBSAG Bull. 100., [30] BBSAG Bull. 102., [31] BBSAG Bull. 103., [32] BBSAG Bull. 104., [33] BBSAG Bull. 105., [34] BBSAG Bull. 107., [35] BBSAG Bull. 108., [36] BBSAG Bull. 110., [37] AAVSO 5., [38] BBSAG Bull. 112. [39] BBSAG Bull. 114. [40] BBSAG Bull. 115., [41] BBSAG Bull. 116., [42] BBSAG Bull. 116. 